\title{
Equilibrium $\boldsymbol{K}$-Shell Excitation of Highly Ionized Neon *
}

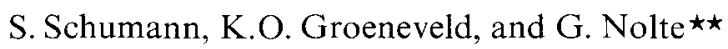 \\ Institut für Kernphysik der Universität Frankfurt am Main, Germany \\ B. Fricke \\ Gesamthochschule Kassel, Germany
}

Received July 28; Revised Version October 11, 1978

\begin{abstract}
Augerelectron emission from foil-excited $\mathrm{Ne}$-ions (6 to $10 \mathrm{MeV}$ beam energy) has been measured. The beam-foil time-of-flight technique has been applied to study electronic transitions of metastable states (delayed spectra) and to determine their lifetimes. To achieve a line identification for the complex structure observed in the prompt spectrum, the spectrum is separated into its isoelectronic parts by an Augerelectron-ion coincidence correlating the emitted electrons and the emitting projectiles of well defined final charge states $q_{f}$. Well resolved spectra were obtained and the lines could be identified using intermediate coupling Dirac-Fock multiconfiguration calculations.

From the total KLL-Augerelectron transition probabilities observed in the electronion coincidence experiment for $\mathrm{Ne}(10 \mathrm{MeV})$ the amount of projectiles with one $K$-hole just behind a $C$-target can be estimated. For foil-excited Ne-projectiles in contrast to single collision results the comparison of transition intensities for individual lines with calculated transition probabilities yields a statistical population of Li- and Be-like configurations.
\end{abstract}

\section{Introduction}

In recent years spectroscopic investigations of the Augerelectron emission after heavy ion-atom collisions have attracted increasing interest [1-5]. The production of $K$-shell vacancies by heavy ion-atom collisions generally is accompanied by multiple $L$-shell ionization. These highly ionized and excited states can be created either by single collisions of fast heavy ions with atoms or molecules of a gaseous target [1-3] or by multiple collisions of projectiles when passing through a solid target $[4,5]$. From these studies one obtains e.g. information about the atomic structure, the excitation mechanism or the population of excited electronic configurations.

In this paper results of a beam-foil experiment are presented.

Kinematic line broadening and the overlap of continuous and discrete electron spectral contributions make a spectral analysis very difficult. To evaluate

\footnotetext{
* Supported by Bundesministerium für Forschung und Technologie * Now at: Phys. Inst. Univ. Heidelberg, Germany
}

the complex electron energy spectra it is necessary to employ further techniques to decompose the observed structures of the foil-excited Ne-Augerelectron spectra; results from two different experimental approaches will be reported.

\section{Experiments}

The Ne-beam of the Frankfurt University $7.5 \mathrm{MV}$ Van de Graaff accelerator has been used to bombard thin carbon foils $\left(5-10 \mu \mathrm{g} / \mathrm{cm}^{2}\right.$ thick). The Auger decay of the post target foil $\mathrm{Ne}$-ions is studied measuring electrons ejected under $42.5^{\circ}$ with respect to the beam direction with a cylindrical mirror analyser [6].

The modified version of this analyser is shown in Fig. 1 schematically. In order to study the post foil $\mathrm{Ne}$-ions as well as the Augerelectron emission, a ring of 6 channel electron multipliers (CEM) placed at the exit cone of the analyser is used to detect the electrons. 


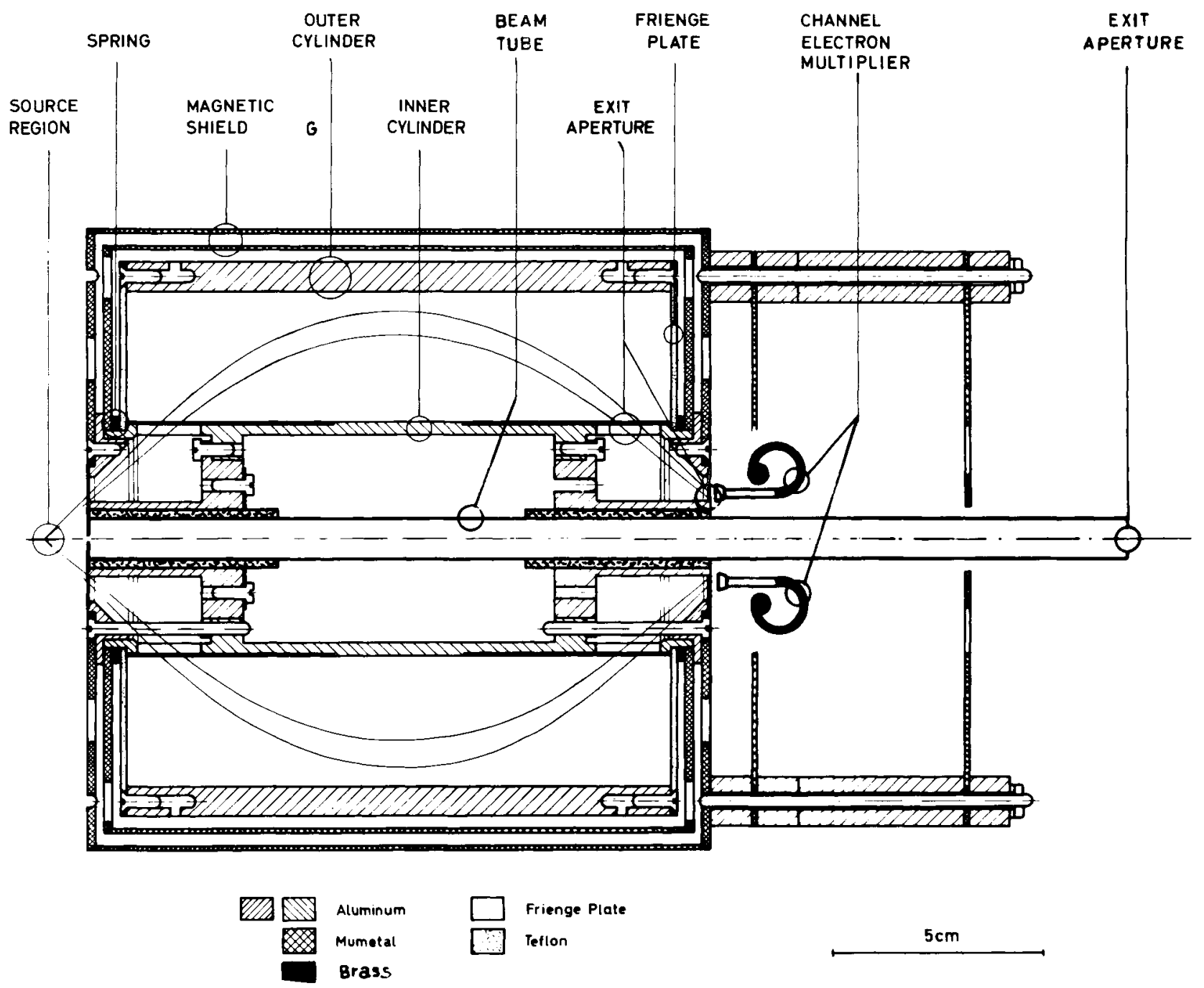

Fig. 1. Scale drawing of the cylindrical mirror analyser

Though this reduces the solid angle of the spectrometer by $40 \%$ it allows e.g. to measure angular distributions ( 11 different angles between $42.5^{\circ}$ and $137.5^{\circ}$ ) by turning the analyser perpendicular to the beam line, to steer the beam through the analyser in order to stop the post foil ions, and to measure the Augerelectrons in coincidence with the outgoing projectiles.

There are different possibilities for line identifications of the observed electron energy spectra:

\subsection{Energy Dependence of the Charge State Distribution}

The charge state distribution of the post foil Ne-ions depends predominantly on the projectile velocity. The attempt to determine the excitation function of $\mathrm{Ne}$ projectiles shows very limited applicability, as under the present experimental conditions energies between 6 and $10 \mathrm{MeV}$ must be chosen to measure electron energy spectra with a reasonable peak to background ratio [7]. In this energy range, however, the change of the excitation function is too small in order to allow any identification. The method has not been persued further.

\subsection{The Beam-Foil Time-of-Flight Technique}

One of the main advantages of a beam-foil arrangement is the possibility to select out metastable states by a time-of-flight technique [8], moving the target foil out of the focal source region of the analyser (see Fig. 1). The energy spectra are then measured as a function of the deexcitation time between the projectile passage through the target foil and the observation of the electron emission at the viewing region of the spectrometer. 


\subsection{The Electron-Ion Coincidence Method}

The projectiles having passed the target foil $(C$, $5-10 \mu \mathrm{g} / \mathrm{cm}^{2}$ ) are spatially separated by the electric field of parallel plates and detected with a suitably collimated surface barrier detector (Fig. 2). Electrons ejected at $42.5^{\circ}$ with respect to the beam direction are analysed by spectrometer and detected by 6 CEM at the exit cone of the analyser (also see Fig. 1). The arrangement allows to measure Augerelectrons decaying in flight in coincidence with outgoing ions of well defined final charge states $[9,10]$.

To process the electronic signals from the detectors standard electronic modules are employed. Two multiscalers are used to obtain a simultaneous measurement of single and coincidence electron energy spectra. The pulse height analyser (PHA) registers the time spectra for each channel of the electron spectra, in order to determine random coincidences correctly. The charge state of the projectiles is measured $26 \mathrm{~cm}$ behind the target foil. With this distance and a pressure of $10^{-6}$ Torr in the vacuum chamber the probability for charge exchange was estimated to be smaller than $0.01 \%$ [11]. For a unique line identification by the

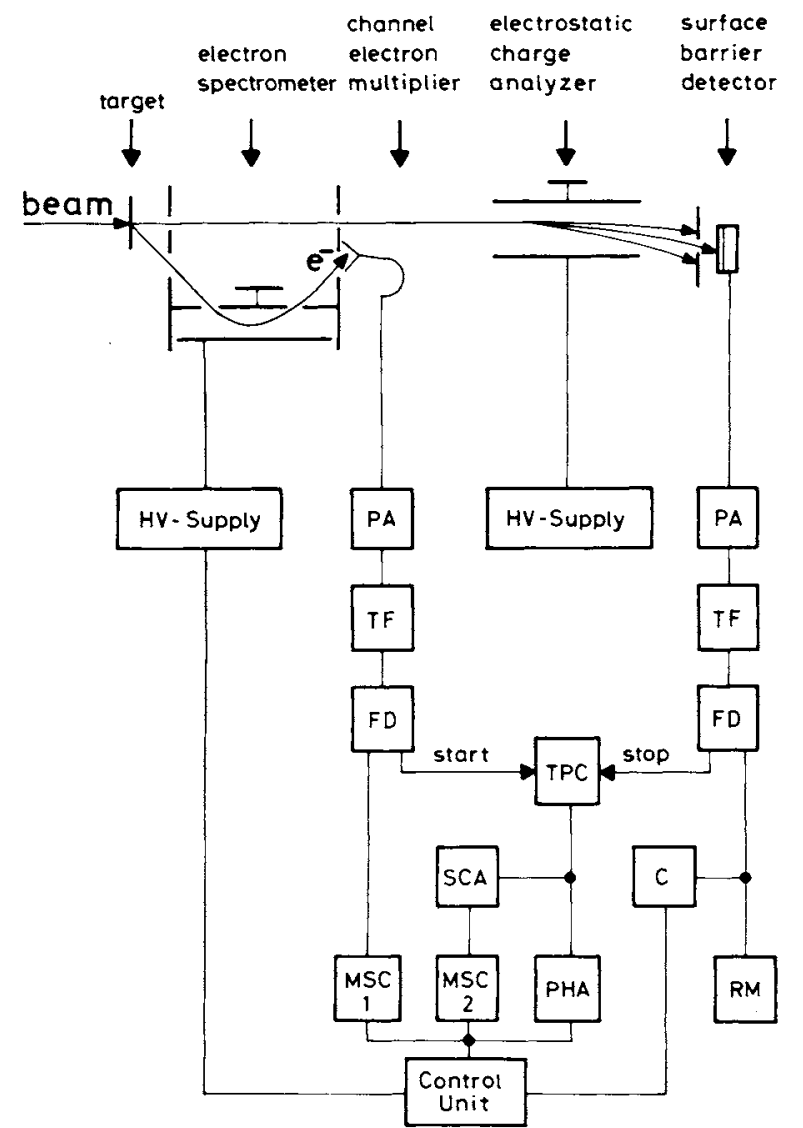

Fig. 2. Block diagram of the electronics including the experimental set-up for the Augerelectron-ion coincidences coincidence measurement different final charge states of the Ne-ions have to be well resolved and post foil charge change collisions must be neglectible small.

\section{Results and Discussion}

\subsection{Line Identification and Lifetime Determination of Metastable States}

Figure 3 demonstrates as a result of the beam-foil timeof-flight technique how the electron energy spectrum of $\mathrm{Ne}(6 \mathrm{MeV})$ changes with separation time $t_{f}$. In the undelayed spectrum (top spectrum, Fig. 3) no individual lines have been resolved. All the lines in the delayed lower spectra may be attributed to the KLL-Auger decay of metastable Li-, Be-, or B-like ${ }^{4} P$-, ${ }^{5} P$ - or ${ }^{6} S$-states. Calculated transition energies obtained from intermediate coupling Dirac-Fock multiconfiguration (MDF) calculations or LS-coupling Hartree-Fock $(\mathrm{HF})$ calculations [12] are indicated by vertical lines. For the most intensive lines from the decrease of the line intensity as a function of the time-of-flight lifetimes of individual metastable states can be determined.

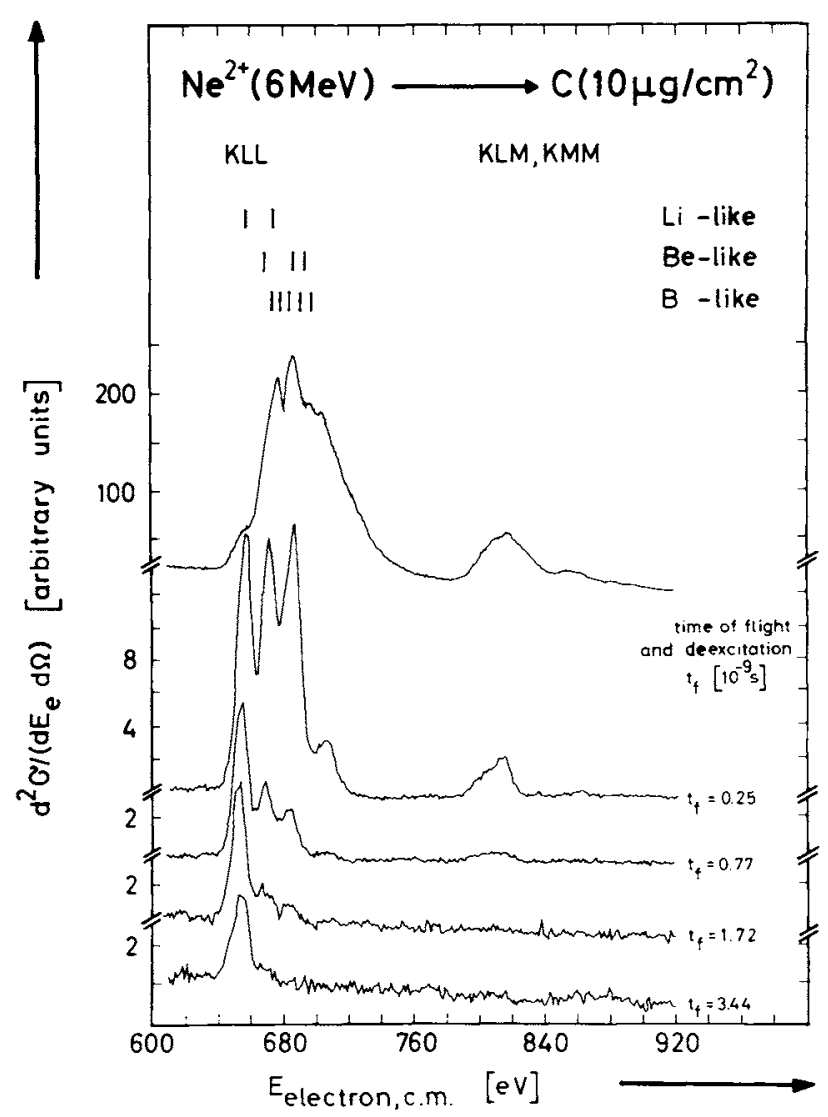

Fig. 3. Augerelectron spectra from beam-foil-excited Ne-projectiles, without delay $t_{f}=0$ ns (top spectrum) and with different delay up to $t_{f}=3.44 \mathrm{~ns}$ 
Table 1. Energies $E$ and lifetimes $t$ of metastable states in the NeKLL-Augerelectron spectrum

\begin{tabular}{|c|c|c|c|c|c|}
\hline \multirow[t]{2}{*}{ Transition } & & \multicolumn{2}{|c|}{ Energy $E(\mathrm{eV})$} & \multicolumn{2}{|c|}{ Life time $t\left(10^{-10} \mathrm{~s}\right)$} \\
\hline & & theor. & exp. & theor. & exp. \\
\hline \multirow[t]{3}{*}{$1 s 2 s 2 p$} & ${ }^{4} P_{1,2}^{0}-{ }^{1} S_{0}{ }^{i}$ & 656.4 & $656 \pm 1$ & $16.45^{b}$ & $16.0 \pm 2.4$ \\
\hline & ${ }^{4} P_{3 ; 2}^{0}-{ }^{1} S_{3}^{\prime \prime}$ & 656.2 & & $6.38^{\circ}$ & $4.0 \pm 1.9$ \\
\hline & ${ }^{4} P_{5}^{0} 2-{ }^{1} S_{0}$ & 656.3 & & $84.06^{a}$ & $104.0 \pm 15.0$ \\
\hline \multirow[t]{3}{*}{$1 s 2 p^{2}$} & ${ }^{4} P_{1 ; 2}^{e}, \ldots{ }^{1} S_{0 ;}^{e^{\prime}}$ & 674.4 & $675 \pm 1$ & $0.94^{b}$ & \\
\hline & ${ }^{4} P_{3}^{e}, \ldots{ }^{1} S_{6} \cdot$ & 673.4 & & $1.70^{b}$ & $1.9 \pm 0.9$ \\
\hline & ${ }^{4} P_{5 ; 2}^{e}-{ }^{1} S_{0}^{r}$ & 673.5 & & $0.28^{b}$ & \\
\hline \multirow[t]{3}{*}{$1 s 2 s 2 p^{2}$} & ${ }^{5} P_{1}^{e^{\prime}}-{ }^{2} P_{12}^{0}=$ & 669.7 & $670 \pm 1$ & $2.12^{b}$ & \\
\hline & ${ }^{5} P_{2}^{e}-{ }^{2} P_{1}^{\prime \prime}=$ & 670.0 & & $4.56^{b}$ & $6.3 \pm 1.8$ \\
\hline & ${ }^{5} P_{3}^{e}-{ }^{2} P_{12}^{0}$ & 670.0 & & $1.14^{b}$ & $1.2 \pm 0.6$ \\
\hline \multirow[t]{3}{*}{$1 s 2 s 2 p^{2}$} & ${ }^{5} P_{1}^{e}-{ }^{2} S_{12}^{c}$ & 685.7 & $686 \pm 1$ & $2.12^{b}$ & \\
\hline & ${ }^{5} P_{2}^{c}-{ }^{2} S_{12}^{c}$ & 686.0 & & $4.56^{b}$ & $6.0 \pm 1.8$ \\
\hline & ${ }^{5} P_{3}^{e}-{ }^{2} S_{12}^{u^{\prime}}$ & 686.0 & & $1.14^{b}$ & $0.9 \pm 0.6$ \\
\hline $1 s 2 p^{3}$ & ${ }^{5} S_{2}^{0}-2 P_{1 / 2}^{0}$ & 693.9 & $694 \pm 1$ & $0.58^{\mathrm{b}}$ & $1.0 \pm 0.6$ \\
\hline \multirow[t]{5}{*}{$1 s 2 s 2 p^{3}$} & ${ }^{6} S^{0}-{ }^{1} S^{c}$ & $671.7^{\circ}$ & & $1.09^{b}$ & \\
\hline & ${ }^{6} S^{0}-{ }^{1} D^{\prime}$ & $678.4^{\circ}$ & & $1.09^{\mathrm{b}}$ & \\
\hline & ${ }^{6} S^{0}-{ }^{3} P^{0}$ & $682.8^{\circ}$ & & $1.09^{b}$ & \\
\hline & ${ }^{6} S^{0}-{ }^{1} P^{0}$ & $690.6^{\circ}$ & $690 \pm 1$ & $1.09^{\mathrm{b}}$ & \\
\hline & ${ }^{6} S^{0}-{ }^{3} P^{0}$ & $697.7^{c}$ & $698 \pm 1$ & $1.09^{b}$ & \\
\hline
\end{tabular}

a From Ref. 13

b From Ref. 14

c From Ref. 12

The results obtained for Ne are summarized in Table 1. There also a comparison is given with calculated transition energies [12] and lifetimes [13-15], showing a very satisfactory agreement between calculated and experimental values for both transition energies and lifetimes. Most of the theoretical values are calculated in the LS-coupling HF-description. The good agreement with the experimental data indicates that even in the case of $\mathrm{Ne}(Z=10)$ the LS-coupling HFdescription is still appropriate.

The measurement of decay curves via the time-of-flight technique allows in some cases to determine lifetimes of substates, even though the corresponding lines cannot be resolved energetically in the spectra. In Fig. 4 such an evaluation is shown for one of the most studied electronic configurations, the Li-like ${ }^{4} P_{5 / 2,3 / 2.1 / 2}^{0}$-states. The data points are obtained from $\mathrm{Ne}(6 \mathrm{MeV})$ on $C$ $\left(8 \mu \mathrm{g} / \mathrm{cm}^{2}\right)$ measurements, partially shown in Fig. 3. The dashed curve gives the sum of the decay of the 3 substates indicated by solid lines.

Although there are only few data points it can be seen from the table in Fig. 4 that the lifetimes obtained agree reasonably well with theoretical predictions [13].

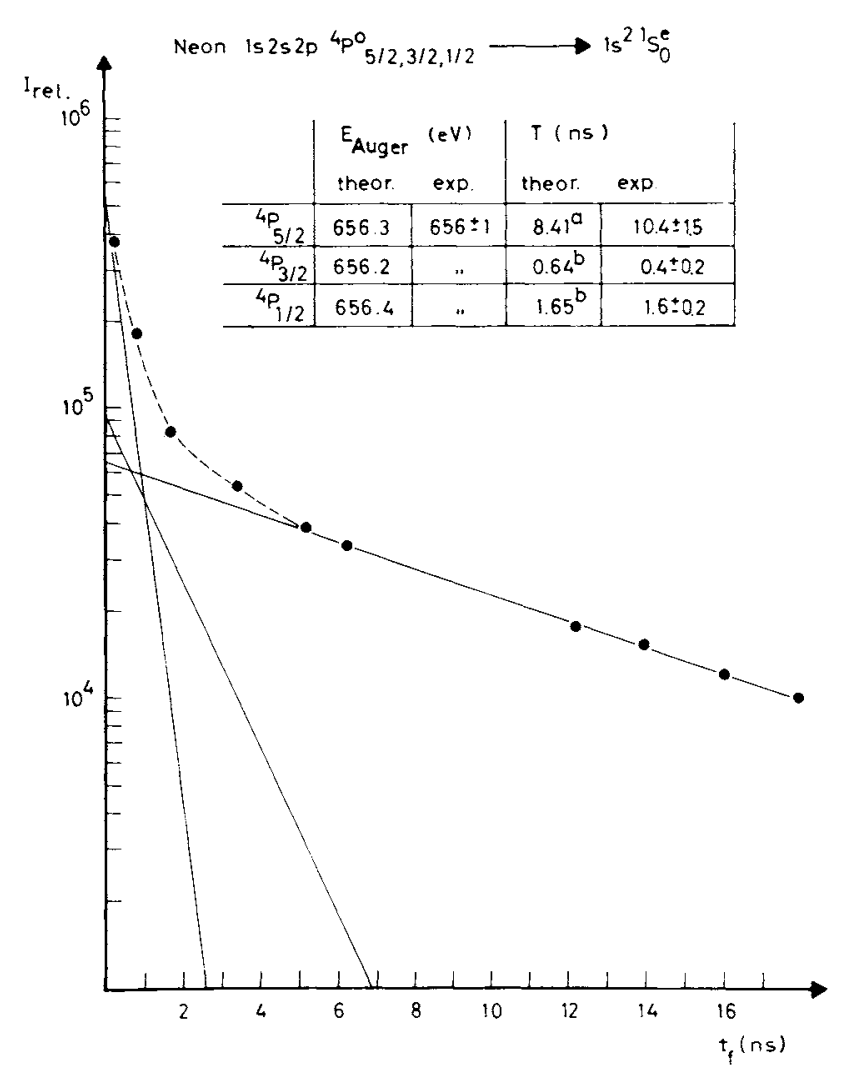

Fig. 4. Measured decay curve for the Li-like ${ }^{4} P^{0}$-states. The included table gives a comparison of the obtained experimental results with theoretical predictions. (Ref. a: [13], Ref. b: [14])

\subsection{Augerelectrons in Coincidence with Ne-Projectiles of Final Charge States $q_{f}=5$ through 8}

The number of electrons involved in the initial electron configuration of the ions just behind the target foil is determined by the measurement of the final charge state $q_{f}$. Assuming an Auger transition the initial charge state $q_{i}$ increases by one $\left(q_{f}=q_{i}+1\right)$.

The result of our coincidence experiment is presented in Fig. 5. There, the foilexcited single KLL-Augerspectrum of $10 \mathrm{MeV} \mathrm{Ne}$ (top spectrum) is compared with the corresponding electron energy spectra measured in coincidence with outgoing Ne-ions having well defined final charge states $q_{f}=5$ through 8 (lower spectra). Also included in Fig. 5 is a sum coincidence spectrum represented by $\bar{q}_{f}=6.6$ which is obtained by adding up the coincidence spectra after weighting with the measured final charge state distribution (compare Fig. 6). To give an idea of the rapidly increasing number of possible electron transitions when more electrons are involved in the initial configurations in Fig. 5 calculated transition energies [12] (also see Table 2) are marked by vertical lines. 


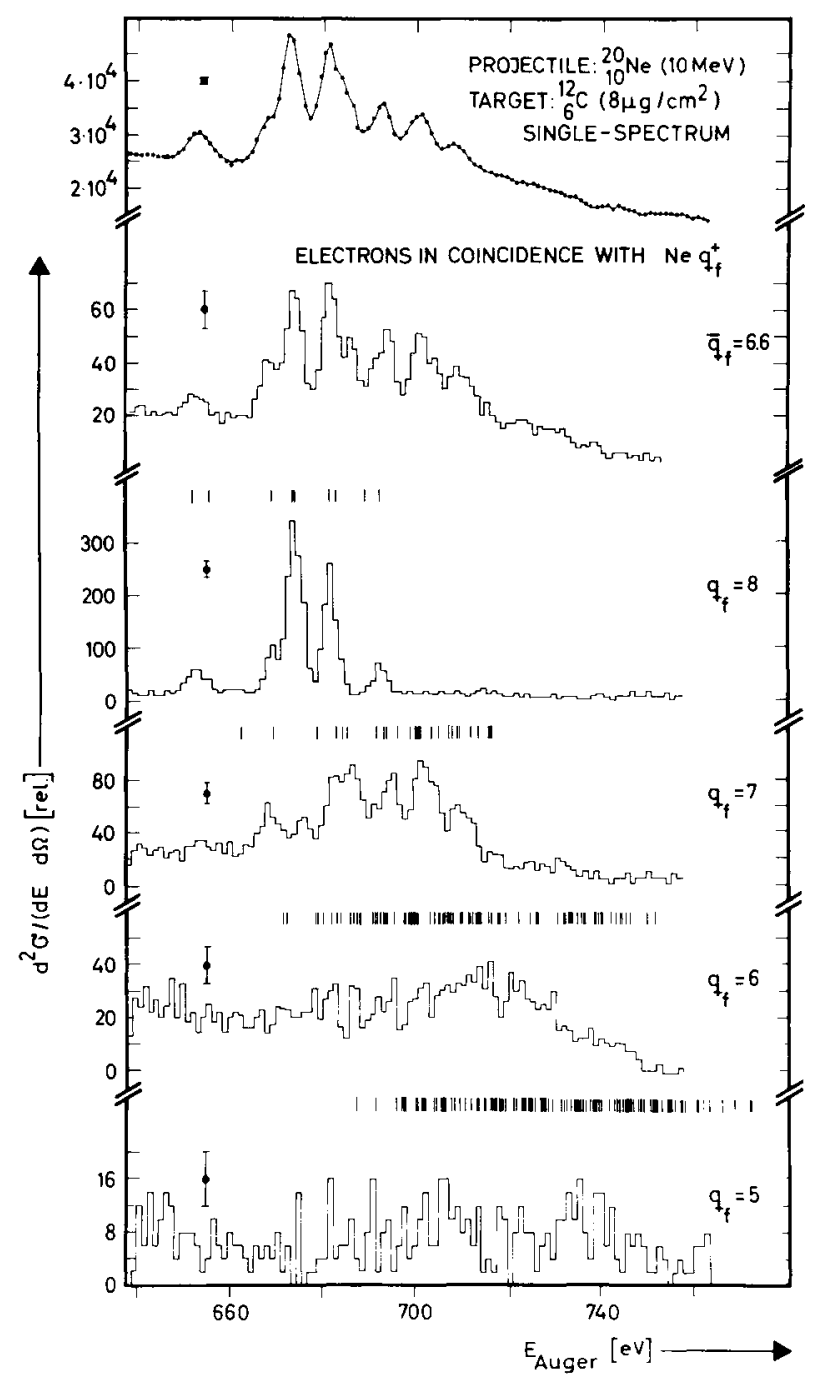

Fig. 5. Augerelectron energy spectra of beam-foil-excited $\mathrm{Ne}(10 \mathrm{MeV}$ incident energy) without coincidence (top spectrum) and in coincidence with projectiles of final charge states $q_{f}=5$ to 8 (bottom spectra). The sum coincidence spectrum $\left(\bar{q}_{f}=6.6\right)$ is obtained from $13.5 \%$ Li-like $\left(q_{f}=8\right)+40.5 \%$ Be-like $\left(q_{f}=7\right)+33.9 \%$ B-like $\left(q_{f}=6\right)$ $+11.9 \%$ C-like $\left(q_{f}=5\right)$ contributions

There have to be considered post-target-foil charge state changing collisions, as there are

1. cascading processes,

2. decay of metastable states downstream the spectrometer viewing region,

3. post target foil collisions with charge exchange.

From the lifetime measurement and the good agreement of the two upper spectra in Fig. 5, however, we find strong evidence that the amount of such charge state changing processes is smaller than $5 \%$ of the total KLL-Augerelectron intensity in the case of foil excited $\mathrm{Ne}$ projectiles with $10 \mathrm{MeV}$ incident energy.
The slightly better energy resolution in the sum spectrum $(\Delta E / E=0.68 \%)$ compared to the single spectrum $(\triangle E / E=0.85 \%$ ) may be explained:

1. By a reduced Doppler broadening [1] caused by accepting only Augerelectrons in coincidence with projectiles scattered into angles $\leqq 0.25^{\circ}$. This restricts the impact parameters to values $\geqq 0.1$ of the Ne $K$-shell radius. Following Taulberg, Briggs, and Vaaben [17] we have estimated the mean impact parameter to be in the order of the $K$-shell radius or even larger. Therefore, it appears that this process contributes very little to the observed difference in line width.

2. By the drastically reduced beam cross section in the case of the coincidence measurements. The Ne-ion flux has to be restricted to values $<50,000 \mathrm{ions} / \mathrm{s}$ in order to detect the ions with a surface barrier detector. This has been achieved by reducing the cross section of the beam from $1 \mathrm{~mm}^{2}$ at $9.5 \cdot 10^{9}$ ions $/ \mathrm{s}(10 \mathrm{nA})$ to $5.3 \cdot 10^{-6} \mathrm{~mm}^{2}$ at $5 \cdot 10^{4}$ ions/s. It appears that this has the dominant influence on the reduction of the line width.

\subsection{K-Vacancy Creation}

The four lower spectra in Fig. 5 are all normalized to the same number $N_{p}$ of Ne-ions detected by the surface barrier detector per channel of the electron energy spectra. It is obvious that the total intensity of the KLL-Augerspectra increases with increasing final charge states. The Li-like KLL-Augerelectron spectrum has nearly twice the total intensity of the Be-like Augerspectrum. This we interpret as, higher stripped Ne-ions do have a larger amount of $K$ vacancies just behind the target foil. Charge state changing effects mentioned above only contribute little $(<5 \%)$ to this increase in KLL-Augerelectron intensity at $10 \mathrm{MeV}$ incident energy. A post-foil one $K$-hole charge state distribution for $\mathrm{Ne}(10 \mathrm{MeV})$ on $C$ $\left(8 \mu \mathrm{g} / \mathrm{cm}^{2}\right)$ is derived from the coincidence spectra shown in Fig. 5. The result is presented in Fig. 6 (full bars). Also included in this figure is the final charge state distribution of $10 \mathrm{MeV}$ Ne measured far behind (27 ns) the target foil (dashed bars).

To obtain the one $K$-vacancy distribution shown in Fig. 6 we have to correct for two different effects:

1. the limited viewing region of the analyser [10] and 2. the different transition probabilities for Li-, Be-, $\mathrm{B}-$, or C-like electronic configurations of $\mathrm{Ne}$ using HF-calculations of Chen and Crasemann [14].

The result is given by dotted bars in Fig. 6 . The amount of He-like Ne-ions $\left(q_{f}=9\right)$ with one $K$-hole is obtained by extrapolation since it cannot be determined from Augerelectron spectroscopy. This procedure seems to 


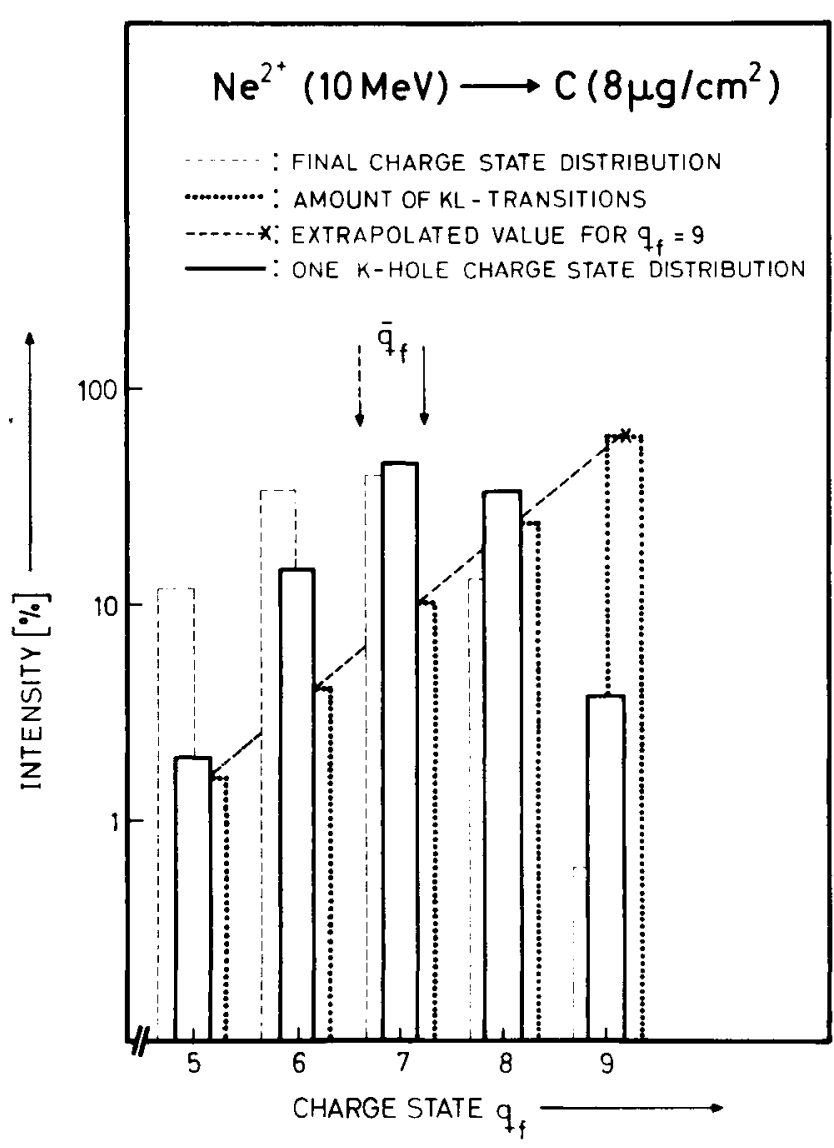

Fig. 6. The one $K$-hole charge state distribution of $10 \mathrm{MeV} \mathrm{Ne}$ just behind a $8 \mu \mathrm{g} / \mathrm{cm}^{2}$ thick C-foil (-) obtained from the measured final charge state distribution (--) and the KL-transition probabilities for different final charge states $(\cdots .$.

be justified since an exponential increase in total KLtransition rates is observed for increasing final charge states.

\subsection{KLM-, KLN-, and Hypersatellite-Transitions}

At least a qualitatively similar behavior of the transition probabilities as a function of the final charge state is observed for the high energetic part of the foil excited $\mathrm{Ne}(10 \mathrm{MeV})$ Augerelectron spectrum, which is populated approximately one order of magnitude less intensively (Fig. 7).

The two top spectra in Fig. 7 - the single $\left(\bar{q}_{f}=6.6\right)$ and the Li-like coincidence spectrum $\left(\bar{q}_{f}=8\right)$ - agree surprisingly well. This finding as well as coincidence measurements of the corresponding Be- and B-like spectra which show only a weak continuous distribution but no individual lines, strongly supports the conclusion that only for the Li-like system $\left(q_{f}=8\right)$ a considerable amount of $K$-holes is created. On the

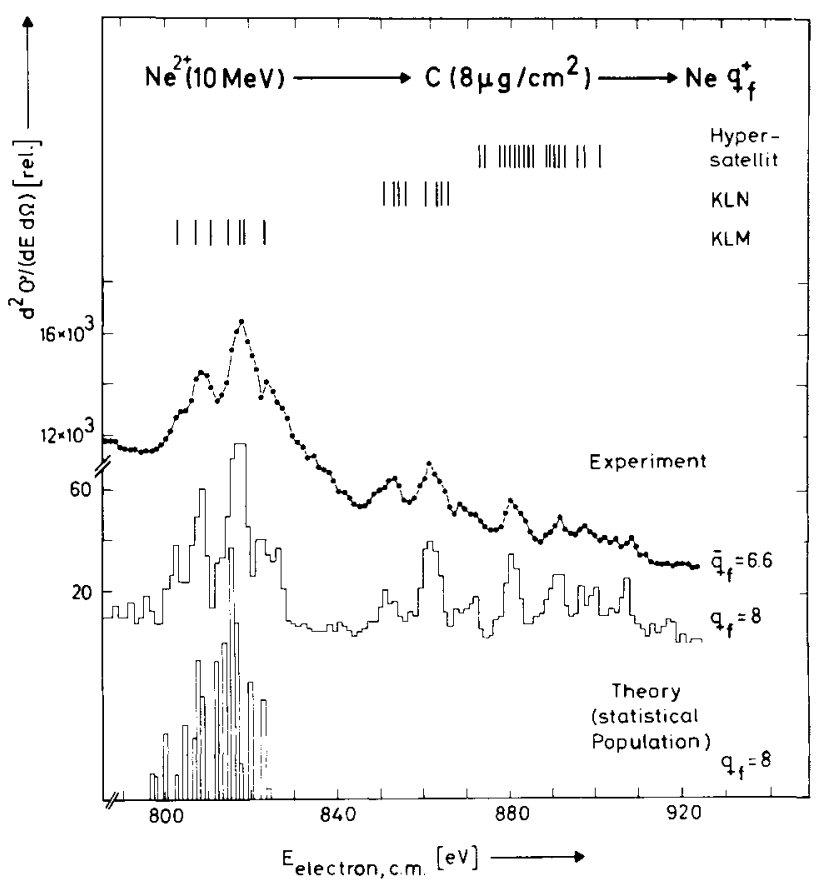

Fig. 7. High-energy Augerelectrons from beam-foil excited $\mathrm{Ne}$ $(10 \mathrm{MeV})$. Middle spectrum: Augerelectrons emitted in coincidence with $\mathrm{Ne}^{+8}$; top spectrum: as above but without coincidence; bottom spectrum: calculated spectrum based on Ref. [16]. The vertical bars indicates some KLM-, KLN- and HypersatelliteAugerelectrons from Table 2

other hand for the Be-like spectrum an enormously large number of possible KLM- or KLN-transitions is imaginable. Thus the observed continuous contribution may be due to many weak discrete Auger transitions which cannot be resolved.

As regards hypersatellite transitions the method of measuring the Auger transitions in coincidence with outgoing ions of different final charge states no longer defines the number of electrons involved in the initial configurations. The final states of hypersatellite transitions decay, except for He-like ones, by a further Augerelectron emission.

In Fig. 7 some of the calculated transition energies are indicated by lines in order to give an idea how to attribute the observed structures. The attempt of a line identification in detail is summarized in Table 2 . In the case of the KLM-Auger transitions of Li-like Ne M.H. Chen [16] has calculated transition probabilities using LS-coupling HF-wavefunctions. From these data the bottom spectrum in Fig. 7 can be constructed assuming statistical population of the initial states and equal probability for creating a $2 s$ - or $2 p$-hole. The good reproduction of the observed structures is a first indication for the validity of the assumptions made. 
Table 2. Energies and intensities of states in beam-foil excited $1 s^{-1}$ - and $1 s^{-2}$-states in $\mathrm{Ne}$

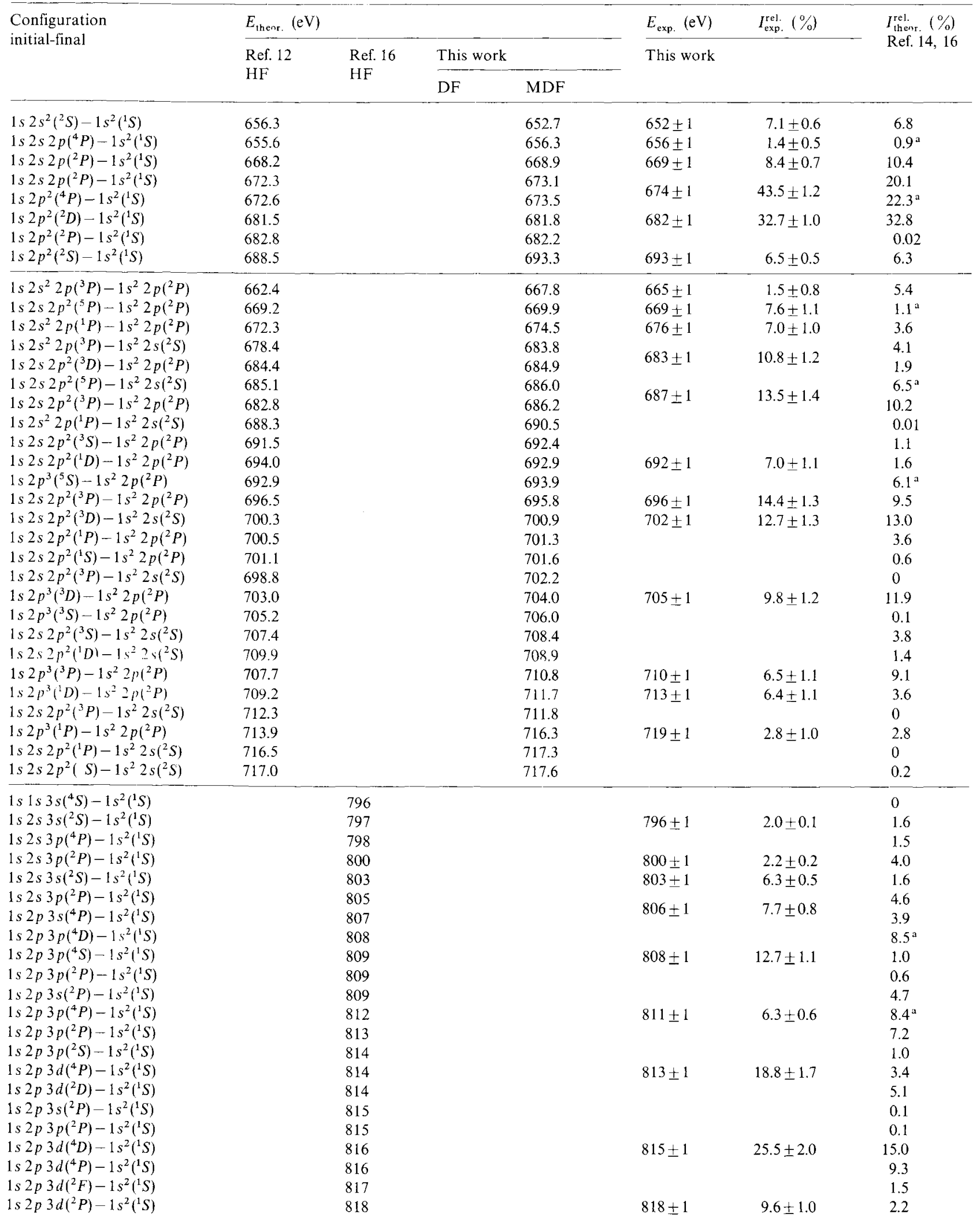

"For the metastable states the incomplete decay during the passage of the electron emitting projectiles through the spectrometer focal region is taken into account 
Table 2 (continued)

\begin{tabular}{|c|c|c|c|c|c|c|c|}
\hline \multirow{3}{*}{$\begin{array}{l}\text { Configuration } \\
\text { initial-final }\end{array}$} & \multicolumn{4}{|c|}{$E_{\text {their. }}(\mathrm{eV})$} & $E_{\text {exp. }}(\mathrm{eV})$ & $I_{\exp .}^{\mathrm{rel}}(\%)$ & \multirow{3}{*}{$\begin{array}{l}I_{\text {thenr. }}^{\text {rel. }}(\%) \\
\text { Ref. } 14,16\end{array}$} \\
\hline & \multirow{2}{*}{$\begin{array}{l}\text { Ref. } 12 \\
\text { HF }\end{array}$} & \multirow{2}{*}{$\begin{array}{l}\text { Ref. } 16 \\
\text { HF }\end{array}$} & This & & \multirow[t]{2}{*}{ This work } & & \\
\hline & & & $\mathrm{DF}$ & MDF & & & \\
\hline $\begin{array}{l}1 s 2 p 3 p\left({ }^{2} D\right)-1 s^{2}\left({ }^{1} S\right) \\
1 s 2 p 3 p\left({ }^{2} S\right)-1 s^{2}\left({ }^{1} S\right) \\
1 s 2 p 3 d\left({ }^{2} F\right)-1 s^{2}\left({ }^{1} S\right) \\
1 s 2 p 3 d\left({ }^{2} D\right)-1 s^{2}\left({ }^{1} S\right) \\
1 s 2 p 3 d\left({ }^{2} P\right)-1 s^{2}\left({ }^{1} S\right)\end{array}$ & & $\begin{array}{l}819 \\
820 \\
823 \\
824 \\
824\end{array}$ & & & $822 \pm 1$ & $8.9 \pm 0.8$ & $\begin{array}{l}6.5 \\
1.2 \\
6.4 \\
0.4 \\
0.4\end{array}$ \\
\hline $\begin{array}{l}1 s 2 s 4 s-1 s^{2} \\
1 s 2 s 4 p-1 s^{2} \\
1 s 2 s 4 d-1 s^{2} \\
1 s 2 s 4 f-1 s^{2} \\
1 s 2 p 4 s-1 s^{2} \\
1 s 2 p 4 p-1 s^{2} \\
1 s 2 p 4 d-1 s^{2} \\
1 s 2 p 4 f-1 s^{2}\end{array}$ & & & $\begin{array}{l}848 \\
850 \\
851 \\
852 \\
858 \\
859 \\
860 \\
861\end{array}$ & & $\begin{array}{l}850 \pm 1 \\
852 \pm 1 \\
860 \pm 1\end{array}$ & & \\
\hline $\begin{array}{l}2 s^{2} 3 s-1 s 2 s \\
2 s 2 p 3 s-1 s 2 p \\
2 s^{2} 3 p-1 s 2 s \\
2 s 2 p 3 p-1 s 2 p\end{array}$ & & & $\begin{array}{l}874 \\
875 \\
878 \\
879\end{array}$ & & $873 \pm 1$ & & \\
\hline $\begin{array}{l}2 s 2 p 3 s-1 s 2 s \\
2 p^{2} 3 s-1 s 2 p \\
2 s^{2} 3 d-1 s 2 s \\
2 s 2 p 3 p-1 s 2 s \\
2 s 2 p 3 d-1 s 2 p \\
2 p^{2} 3 p-1 s 2 p \\
2 s 2 p 3 d-1 s 2 s \\
2 p^{2} 3 d-1 s 2 p \\
2 s 2 p 3 s-1 s 2 p\end{array}$ & & & $\begin{array}{l}880 \\
882 \\
884 \\
884 \\
885 \\
886 \\
890 \\
891 \\
892\end{array}$ & & $891 \pm 1$ & & \\
\hline
\end{tabular}

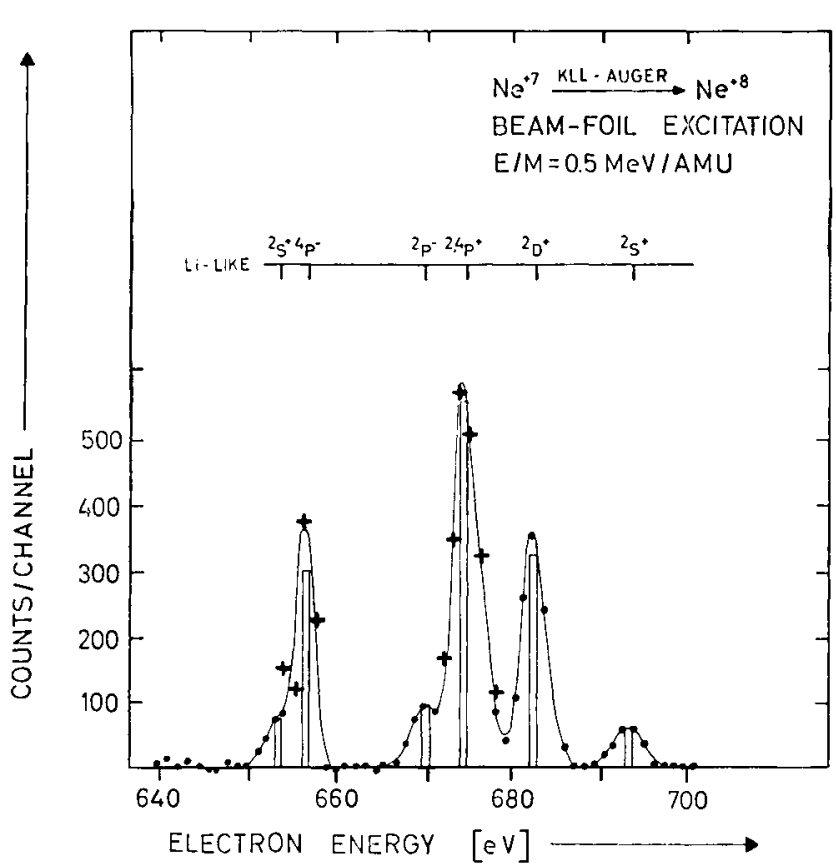

Fig. 8. KLL-Augerelectrons from Li-like Ne. The points and crosses (corrected for metastability, see text; error bar 20\%) are measured data. The solid line is the sum to least square fits to the six peaks observed. The vertical bars are the theoretical intensities. The initial configuration is given above the calculated energies (vertical lines)

\subsection{The Li-, and Be-like KLL-Auger Spectra}

The Li-like Auger spectrum $\left(q_{i}=7\right)$ is the most simple of the Auger spectra measured in coincidence with outgoing projectiles $\left(q_{f}=8\right)$. The comparatively simple structure of the spectrum (Fig. 8) is readily understood: for three electrons $\left(q_{i}=7 ; Z=10\right)$ involved in the initial configuration 8 different excited initial electronic configurations can be deexcited via KLL-Augerelectron emission. If there are 4 electrons (Be-like) involved, this number increases to 26 possible different excited initial electronic configurations (compare Fig. 9).

Transition energies calculated from Dirac-Fock wavefunctions $[7,18]$ are marked in Figs. 8 and 9 by vertical bars (see also Table 2). The agreement with the experimental electron energies in the center of mass frame is excellent.

In Fig. 8 a comparison of the intensities is also included. A computer fit program [19] was applied to determine the relative double differential cross sections from the experimental data; the dashed lines in Figs. 8 and 9 are fits of Gaußian shape for single lines, the sum is indicated as solid curve. The background originates from knock-on-electron contributions with continuous energy distribution [5]. 


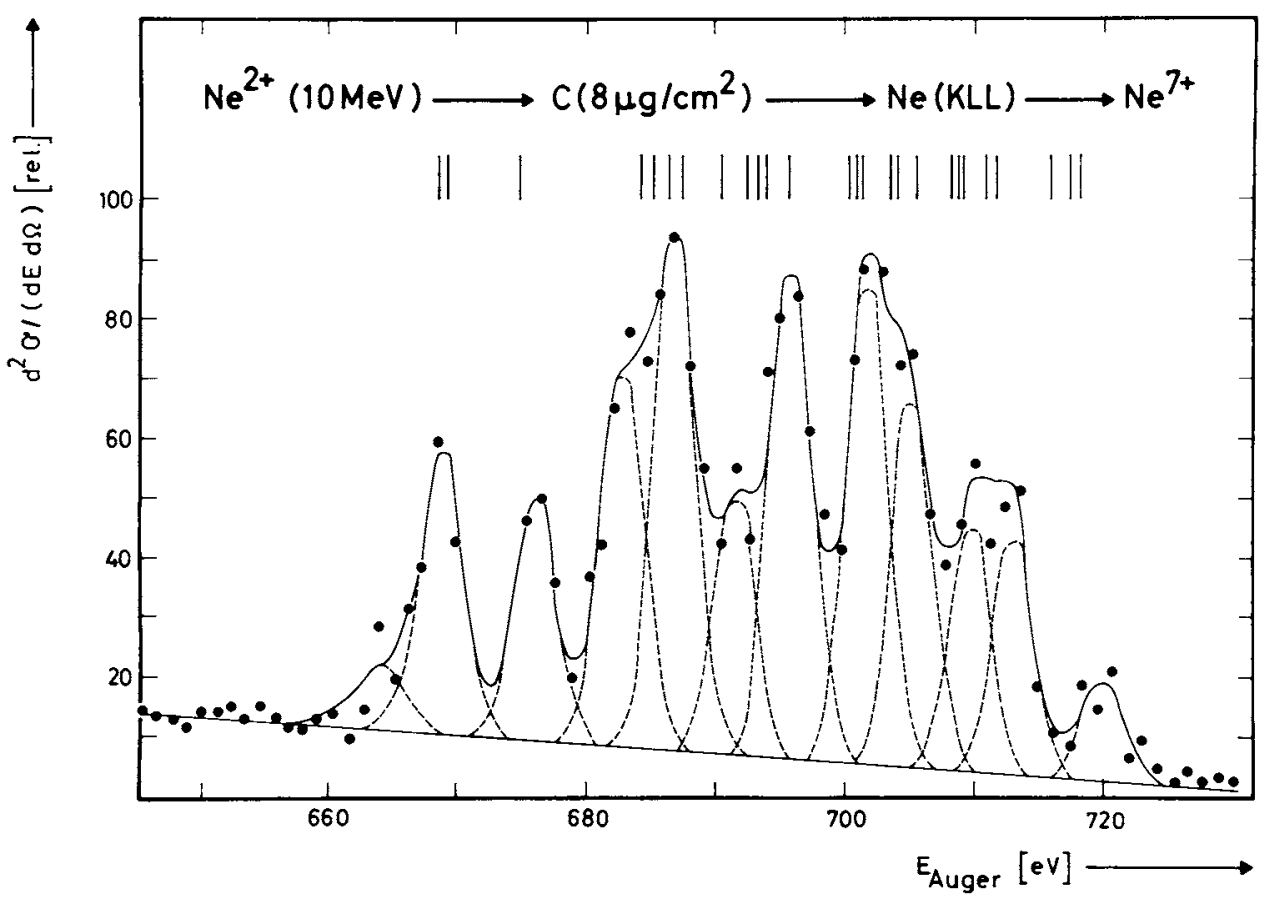

Fig. 9. KLL-Augerelectrons from Be-like Ne. The dashed lines are least square fits to twelve peaks. The solid line is the sum of the least square fits to the structure observed. The calculated energies are given by vertical lines

As already mentioned before, to compare the measured relative line intensities with theoretical transition rates obtained from LS-coupling HF-calculations $[14,15$, 17] two important assumptions have to be made [10]: 1. statistical population of the initial configuration, 2. equal probability for creating a $2 s$ or $2 p$ vacancy. However, for a comparison also the lifetime of the populated states has to be considered. Only the metastable states (compare Table 1) do not decay completely during the time while the electron emitting projectiles pass through the spectrometer viewing region $(\Delta l=0.6 \mathrm{~mm})$. From the projectile velocity $v_{p}$ the corresponding observation time interval can be determined to $\Delta t=5 \cdot 10^{-11} \mathrm{~s}$. For metastable states with lifetime $\tau$ only a fraction $f(\Delta t, \tau)=-\exp (-\Delta t / \tau)$ of the complete decay is observed [10]. This correction for the two metastable ${ }^{4} P$-states made and the assumptions yield the calculated Li-like KLL-Auger spectrum indicated by bars in Fig. 8. When normalizing to the same total KLL-Augerelectron transition probability very good agreement of the measured and calculated $[14,15]$ Li-like Auger spectra is obtained. This result allows the important conclusion: The multicollisionbeam-foil excitation of the studied projectiles yields a statistical population of the initial configurations. Also the Be-like KLL-spectrum (Fig. 9) and the Li-like KLM-spectrum (Fig. 7) support this conclusion. In contrast to this finding Matthews et al. [20] reported results from a single collision $45 \mathrm{MeV} \mathrm{Cl}^{13+}$ on $\mathrm{Ne}$ gas experiment, where they observe a strong overpopulation of Li-like $1 s 2 s^{2}$ - and $1 s 2 s 2 p$-states relative to the $1 s 2 p^{2}$-configurations. Similar conclusions can be drawn from $56 \mathrm{MeV}$ Ar or $200 \mathrm{MeV} \mathrm{Xe}$ on Ne $[21,22]$ data.

\section{Conclusions}

Two experimental techniques to illucidate the complex structures observed in a multicollision beam-foil experiment have been presented.

First, for metastable configurations of foil excited $\mathrm{Ne}$ Auger transition energies and lifetimes have been determined. Good agreement is found for both lifetimes $\tau$ and transition energies $E_{A}$ between the measured values and MDF- or HF-calculations.

Second, the Augerelectron-ion coincidence experiment for the first time allows to attribute most of the lines observed in the prompt spectrum of foil excited $\mathrm{Ne}$ to well defined charge states. The total KL-transition rates show an exponential increase for the creation of one $K$-hole with increasing charge state of the Neprojectiles. The comparison of relative line intensities with calculated transition probabilities yields the con- 
clusion that Li- and Be-like configurations which decay by KLL- or KLM-Auger transitions are populated statistically by beam-foil excitation.

The authors appreciate M.H. Chen's and B. Crasemann's valuable calculations and thank them, P. Armbruster, C.P. Bhalla, and Gy. Szabó for stimulating comments and discussions.

\section{References}

1. Stolterfoht, N.: In: Topics in Current Physics, Sellin, I.A. (ed.). Berlin-Heidelberg-New York: Springer 1978

2. Matthews, D.L.: Methods of Experimental Physics-Accelerators in Atomic Physics, Richard, P. (ed.). New York: Academic Press 1977

3. Woods, C.W., Kauffman, R.L., Jamison, K.A., Stolterfoht, N., Richard, P.: Phys. Rev. A 13, 1358 (1976)

4. Sellin, I.A.: In: Topics in Current Physics, Sellin, I.A. (ed.). Berlin-Heidelberg-New York: Springer 1978

5. Groeneveld, K.O.: In: Beamfoil Spectroscopy, Sellin, I.A., Pegg, D.J. (eds.). New York: Plenum 1976

6. Dietz, E., Groeneveld, K.O., Spohr, R., Staudte, R.: Nucl. Instr. Meth. 105, 467 (1972)

7. Groeneveld, K.O., Mann, R., Nolte, G., Schumann, S., Spohr, R., Fricke, B.: Z. Physik A 274, 191 (1975)

8. Groeneveld, K.O., Mann, R., Nolte, G., Schumann, S., Spohr, R.: Phys. Lett. 54 A, 335 (1975)

9. Groeneveld, K.O., Nolte, G., Schumann, S., Sevier, K.D.: Phys. Lett. 56 A, 29 (1976)

10. Schumann, S., Groeneveld, K.O., Sevier, K.D., Fricke, B.: Phys. Lett. $60 \mathrm{~A}, 289$ (1977)

11. Betz, H.-D.: Rev. Mod. Phys. 44, 465 (1972)
12. Matthews, D.L., Johnson, B.M., Moore, C.F.: Atomic Data and Nucl. Data Tables 15, 41 (1975)

13. Cheng, K.T., Lin, C.P., Johnson, W.R.: Phys. Lett. 48 A, 437 (1974); for detailed analysis see also: Dohmann, H.D., Liesen, D., Pfeng, E.: Z. Physik A 285, 171 (1978)

14. Chen, M.H., Crasemann, B.: Phys. Rev. A 12, 959 (1975)

15. Bhalla, C.P.: Phys. Rev. A 12, 122 (1975)

16. Chen, M.H.: Phys. Rev. A 15, 2318 (1977)

17. Taulberg, K., Briggs, J.S., Vaaben, J.: J. Phys. B 9, 1351 (1976)

18. Desclaux, J.P.: Comp. Phys. Comm. 9, 31 (1975)

19. Comfort, J.R.: ANL, Informal Report PHY - 1970B (1970)

20. Matthews, D.L., Fortner, R.J., Schneider, D., Moore, C.F.: Phys. Rev. A 14, 1561 (1976)

21. Mann, R., Folkmann, F., Groeneveld, K.O.: Phys. Rev. Lett. 37, 1674 (1976)

22. Stolterfoht, N., Schneider, D., Mann, R., Folkmann, F.: Xth Intern. Conf. on Physics of Electronic and Atomic Collisions 1977, Paris

S. Schumann

K.O. Groeneveld

G. Nolte

Institut für Kernphysik

Universität Frankfurt/Main

August-Euler-Straße 6

D-6000 Frankfurt/Main 90

Federal Republic of Germany

B. Fricke

Theoretische Physik

Gesamthochschule Kassel

Heinrich-Plett-Straße 40

D-3500 Kassel

Federal Republic of Germany 